\title{
STRUCTURAL COMPLETENESS IN RELEVANCE LOGICS
}

\author{
J.G. RAFTERY AND K. ŚWIRYDOWICZ
}

\begin{abstract}
It is proved that the relevance logic $\mathbf{R}$ (without sentential constants) has no structurally complete consistent axiomatic extension, except for classical propositional logic. In fact, no other such extension is even passively structurally complete.
\end{abstract}

\section{INTRODUCTION}

For present purposes, a logic is a substitution-invariant finitary consequence relation $\vdash$ over sentential formulas in an algebraic signature, cf. $[3,7,13]$. Every such $\vdash$ is the deducibility relation $\vdash_{\mathbf{F}}$ of a formal system F comprising axioms and finite inference rules. Thus, $\vdash$ consists of rules of derivation $\Gamma / \alpha$ and is not determined by its theorems alone. An extension of $\vdash_{\mathbf{F}}$ is a superset that is itself a logic in the same signature. It is an axiomatic extension if it has the form $\vdash_{\mathbf{F}^{\prime}}$, where $\mathbf{F}^{\prime}$ adds only axioms (not inference rules) to $\mathbf{F}$. We tend to use $\mathbf{F}$ and $\vdash_{\mathbf{F}}$ interchangeably below.

A logic $\vdash$ is structurally complete if each of its proper extensions has some new theorem (as opposed to having nothing but new rules of derivation). Equivalently, $\vdash$ is structurally complete if it contains all of its admissible rules - these are the finite schematic rules under which its set of theorems is closed. In this case, $\vdash$ has a high degree of self-sufficiency in relation to its meta-theory. Much of the literature on admissibility and structural completeness deals with systems possessing the weakening axiom $p \rightarrow(q \rightarrow p)$; see [24] and the references in [6]. Here, however, we are concerned with the family of relevance logics, where less is known about these features.

The relevance logic $\mathbf{R}$ of Anderson and Belnap is formulated without sentential constants in [1], for the sake of a variable-sharing principle. Its conservative expansion $\mathbf{R}^{\mathbf{t}}$ incorporates the 'Ackermann truth constant' $\mathbf{t}$, and counts among the substructural logics of [15]. The adoption of $\mathbf{t}$ is innocuous for most purposes, but it makes a considerable difference to the lattice of axiomatic extensions and to questions of structural completeness.

For instance, classical propositional logic $(\mathbf{C P L})$ is the largest consistent extension of $\mathbf{R}$, and $\mathbf{t}$ is definable in $\mathbf{C P L}$ as $p \rightarrow p$, but $\mathbf{R}^{\mathbf{t}}$ has axiomatic extensions incomparable with $\mathbf{C P L}$. Moreover, infinitely many of these are

Key words and phrases. Admissible rule, structurally complete, relevance logic.

The first author was supported in part by the National Research Foundation of South Africa (UID 85407). 
structurally complete (see the first remark after Theorem 4). In contrast, we prove here that $\mathbf{R}$ has no structurally complete consistent axiomatic extension, other than CPL. Apart from highlighting the impact of $\mathbf{t}$, this complements negative results about neighbouring systems in [22, Sec. 8, 9], whose own proofs break down in the context of $\mathbf{R}$.

\section{The Algebras}

A De Morgan monoid $\boldsymbol{A}=\langle A ; \cdot, \rightarrow, \wedge, \vee, \neg, \mathbf{t}\rangle$ comprises a distributive lattice $\langle A ; \wedge, \vee\rangle$, a commutative monoid $\langle A ; \cdot, \mathbf{t}\rangle$ satisfying $x \leqslant x \cdot x$ (where $\leqslant$ is the lattice order), a unary operation $\neg$ on $A$ satisfying $\neg \neg x=x$ and

$$
x \cdot y \leqslant z \Longrightarrow \neg z \cdot y \leqslant \neg x,
$$

and a binary operation $\rightarrow$ on $A$ satisfying $x \rightarrow y=\neg(x \cdot \neg y)$. It follows that $\leqslant$ is compatible with $\cdot$, that $\neg$ is an anti-automorphism of $\langle A ; \wedge, \vee\rangle$, and that $\boldsymbol{A}$ satisfies

$$
\mathbf{t} \leqslant x \Longleftrightarrow x \rightarrow x \leqslant x
$$

see [1]. As $\cdot$ and $\rightarrow$ are inter-definable, either one could be eliminated. We abbreviate $(x \rightarrow y) \wedge(y \rightarrow x)$ as $x \leftrightarrow y$.

The $\mathbf{t}$-free subreducts of De Morgan monoids $\boldsymbol{A}$ (i.e., the subalgebras of the reducts $\langle A ; \cdot, \rightarrow, \wedge, \vee, \neg\rangle)$ are called relevant algebras.

De Morgan monoids and relevant algebras form varieties DM and RA, respectively. A finite equational basis for RA is given in [14]; also see [12] and [18, Cor. 4.11]. A finitely generated relevant algebra $\boldsymbol{A}$ has a least and a greatest element, which form a Boolean subalgebra [26, Prop. 5], and $\boldsymbol{A}$ itself is a reduct - not merely a subreduct - of a De Morgan monoid. The unique neutral element for $\cdot$ is the greatest lower bound of all $a \rightarrow a$, where $a$ ranges over any finite generating set for $\boldsymbol{A}$; see for instance [22, Thm. 5.3].

For each subquasivariety $\mathrm{K}$ of $\mathrm{DM}$, there is a logic $\vdash^{\mathrm{K}}$ with the same signature, defined as follows: for any set $\Gamma \cup\{\alpha\}$ of formulas, $\Gamma \vdash^{\mathrm{K}} \alpha$ iff there exist $\gamma_{1}, \ldots, \gamma_{n} \in \Gamma$ such that every algebra in $\mathrm{K}$ satisfies

$$
\mathbf{t} \leqslant \gamma_{1} \wedge \ldots \wedge \gamma_{n} \Longrightarrow \mathbf{t} \leqslant \alpha .
$$

Because DM satisfies $\mathbf{t} \leqslant x \leftrightarrow y \Longleftrightarrow x=y$, the logic $\vdash^{\mathrm{K}}$ is algebraizable in the sense of [3], with $\mathrm{K}$ as its unique equivalent quasivariety. The map $\mathrm{K} \mapsto \vdash^{\mathrm{K}}$ is a lattice anti-isomorphism from the subquasivarieties of DM to the extensions of the relevance logic $\mathbf{R}^{\mathbf{t}}$, carrying the subvarieties of DM onto the axiomatic extensions. In particular, $\mathbf{R}^{\mathbf{t}}$ is algebraized by DM (cf. $[9,10]$ and Dunn's contributions to [1]).

The logic $\mathbf{R}$ lacks the symbol $\mathbf{t}$, but all claims in the previous paragraph remain true when we replace $\mathbf{R}^{\mathbf{t}}$ by $\mathbf{R}$, and DM by RA, provided we use (1) to eliminate all mention of $\mathbf{t}$.

The following theorem, suggested by [2], is proved in [22, Sec. 7]. 
Theorem 1. A logic algebraized by a variety $\mathrm{K}$ is structurally complete iff every proper subquasivariety of $\mathrm{K}$ generates a proper subvariety of $\mathrm{K}$.

As the 2-element Boolean algebra 2 embeds into every finitely generated nontrivial relevant algebra, the variety BA of Boolean algebras is the smallest nontrivial subquasivariety of RA, i.e., CPL is the largest consistent extension of $\mathbf{R}$.

In [25], the second author showed that, in the lattice of axiomatic extensions of $\mathbf{R}$, the unique co-atom $\mathbf{C P L}$ covers just three logics. This is Theorem 2 below; it is the key to our main result, Theorem 4. Its statement involves three simple relevant algebras $\boldsymbol{A}_{1}, \boldsymbol{A}_{2}$ and $\boldsymbol{S}_{3}$ (the last being the 3 -element Sugihara algebra). Their Hasse diagrams are as depicted.

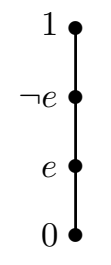

$\boldsymbol{A}_{1}$

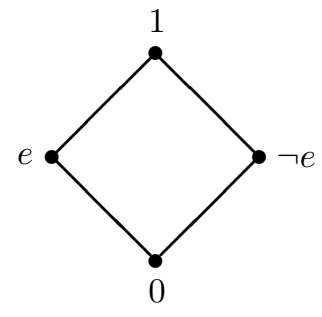

$\boldsymbol{A}_{2}$

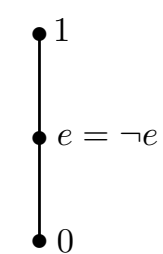

$S_{3}$

In each case, $a \cdot 0=0$ for all elements $a$, while $e$ is the (undistinguished) neutral element for $\cdot$; its image under $\neg$ is indicated. In $\boldsymbol{A}_{1}$ and $\boldsymbol{A}_{2}$, we have $\neg e \cdot \neg e=1$. Our notation makes $\rightarrow$ the same set-theoretic operation on $\boldsymbol{A}_{1}$ as on $\boldsymbol{A}_{2}$. The tables for $\rightarrow$ are as follows.

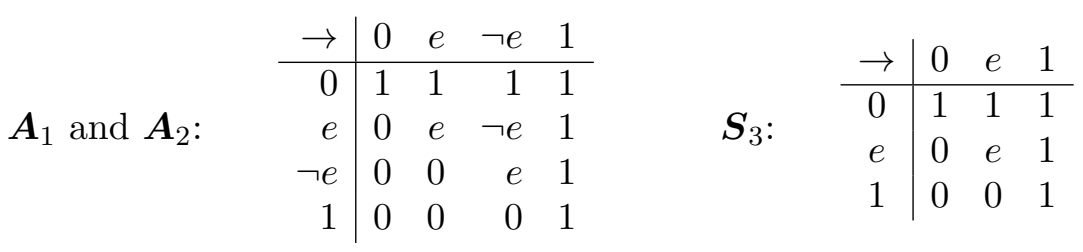

As usual, $\mathrm{V}(\boldsymbol{A})$ and $\mathrm{Q}(\boldsymbol{A})$ denote, respectively, the variety and the quasivariety generated by a single algebra $\boldsymbol{A}$.

Theorem 2. (Świrydowicz [25]) In the lattice of varieties of relevant algebras, the covers of BA are just $\mathrm{V}\left(\boldsymbol{A}_{1}\right), \mathrm{V}\left(\boldsymbol{A}_{2}\right)$ and $\mathrm{V}\left(\boldsymbol{S}_{3}\right)$.

In other words, for each axiomatic extension of $\mathbf{R}$, strictly weaker than $\mathbf{C P L}$, there exists $\boldsymbol{B} \in\left\{\boldsymbol{A}_{1}, \boldsymbol{A}_{2}, \boldsymbol{S}_{3}\right\}$ such that the theorems of the extension all take values $\geqslant e$ on any interpretation of their variables in $\boldsymbol{B}$.

The following well-known result will be needed below; a stronger statement is proved in [8, Lem. 1.5].

Lemma 3. For any algebra $\boldsymbol{C}$, each subdirectly irreducible member of $\mathrm{Q}(\boldsymbol{C})$ can be embedded into an ultrapower of $\boldsymbol{C}$. 


\section{Structural InCOMPleteness}

Meyer and Dunn [20] proved that the disjunctive syllogism $p, \neg p \vee q / q$ is admissible in $\mathbf{R}$. It does not belong to $\vdash_{\mathbf{R}}$ (take $p=e$ and $q=0$ in $\boldsymbol{A}_{1}$ or $\boldsymbol{S}_{3}$ ). Therefore, $\mathbf{R}$ is not structurally complete. We can now show that the same applies to almost all axiomatic extensions of $\mathbf{R}$.

Theorem 4. No consistent axiomatic extension of $\mathbf{R}$ is structurally complete, except for $\mathbf{C P L}$.

Proof. Let $\mathrm{K}$ be a variety of relevant algebras, including some non-Boolean algebras. Then $\mathrm{K}=\mathrm{V}(\boldsymbol{A})$, where $\boldsymbol{A}$ is a free $\aleph_{0}$-generated algebra in $\mathrm{K}$. By Theorem 2, K includes an algebra $\boldsymbol{B}$ that is one of $\boldsymbol{A}_{1}, \boldsymbol{A}_{2}, \boldsymbol{S}_{3}$. Of course, $\mathbf{2} \in \mathrm{K}$, so $\boldsymbol{C}:=\boldsymbol{A} \times \boldsymbol{B} \times \mathbf{2} \in \mathrm{K}$. Because $\mathrm{V}(\boldsymbol{C})$ is closed under homomorphic images, $\boldsymbol{A} \in \mathrm{V}(\boldsymbol{C})$, whence $\mathrm{K}=\mathrm{V}(\boldsymbol{C})$.

We claim that $\mathrm{K} \neq \mathrm{Q}(\boldsymbol{C})$. This will follow if $\boldsymbol{B} \notin \mathrm{Q}(\boldsymbol{C})$. As $\boldsymbol{B}$ is simple, it suffices, by Lemma 3, to show that $\boldsymbol{B}$ can't be embedded into an ultrapower of $\boldsymbol{C}$. But $\boldsymbol{B}$ is a finite algebra of finite type, so the attribute of lacking a subalgebra isomorphic to $\boldsymbol{B}$ is first order definable. Thus, by Łos' Theorem [4, Thm. V.2.9], $\boldsymbol{B}$ won't embed into an ultrapower of $\boldsymbol{C}$ unless it embeds into $\boldsymbol{C}$ itself. To see that $\boldsymbol{B}$ can't be embedded into $\boldsymbol{C}$, it suffices to find an existential first order sentence that holds in $\boldsymbol{B}$ and fails in $\boldsymbol{C}$. Let $\Phi$ be the existential positive sentence

$$
\exists x(x \rightarrow x=x \quad \& \quad \neg x \rightarrow x \leqslant \neg x)
$$

(where $\alpha \leqslant \beta$ abbreviates $\alpha \wedge \beta=\alpha$ ). Then $\Phi$ is true in $\boldsymbol{B}$, since $e$ can serve as $x$ in all three possible cases. But, like any positive sentence, $\Phi$ persists under homomorphisms, so it fails in $\boldsymbol{C}$, because it clearly fails in $\mathbf{2}$.

This vindicates the claim that $\mathrm{Q}(\boldsymbol{C}) \neq \mathrm{K}=\mathrm{V}(\boldsymbol{C})$. Thus, $\mathrm{Q}(\boldsymbol{C})$ is a proper subquasivariety of $\mathrm{K}$ which fails to generate a proper subvariety of K. That completes the proof, in view of Theorem 1.

Three remarks are in order:

First, as we mentioned in the introduction, the situation for $\mathbf{R}^{\mathbf{t}}$ is entirely different. One reason is that the simple De Morgan monoid $\left\langle\boldsymbol{S}_{3}, e\right\rangle$ has no 2element subalgebra (unlike $\boldsymbol{S}_{3}$ ). In fact, $\left\langle\boldsymbol{S}_{3}, e\right\rangle$ generates a variety excluding 2, which is minimal as a quasivariety [22, Footnote 8, p. 489]. Moreover, the logic algebraized by $\mathrm{V}\left(\left\langle\boldsymbol{S}_{3}, e\right\rangle\right)$ contains infinitely many structurally complete axiomatic extensions of $\mathbf{R}^{\mathrm{t}}$. Indeed, in the subvariety lattice of DM, the nontrivial varieties of odd Sugihara monoids (i.e., De Morgan monoids satisfying $x \cdot x=x$ and $\mathbf{t}=\neg \mathbf{t}$ ) form a chain of order type $\omega+1$, ascending from $\mathrm{V}\left(\left\langle\boldsymbol{S}_{3}, e\right\rangle\right)$. And the corresponding logics are structurally complete, by Theorem 1, because every quasivariety of odd Sugihara monoids is a variety - see for instance Theorem 7.3, Fact 7.6 and page 2190 of [16] (or stronger results in $[21,17])$. 
Secondly, the word 'axiomatic' cannot be dropped in Theorem 4. Indeed, every logic has a unique structurally complete extension with the same set of theorems [19]. (It is obtained by treating admissible rules as new inference rules.) In the case of $\mathbf{R}$, this extension is strictly weaker than $\mathbf{C P L}$, as $\mathbf{C P L}$ has more theorems than $\mathbf{R}$.

Thirdly, an admissible rule $\gamma_{1}, \ldots, \gamma_{n} / \alpha$ of a logic $\vdash$ is said to be passive if no substitution turns all of $\gamma_{1}, \ldots, \gamma_{n}$ into theorems of $\vdash$. If $\vdash$ contains all of its passive admissible rules, it is said to be passively structurally complete $[5,11]$, or overflow complete. The following characterization is essentially due to Wroński [27]. For varieties K, its forward implication is implicit in Bergman [2]. (These two papers eschew explicit connections with logic, but a generalization of Theorem 5 is proved in [23, Sec. 7].)

Theorem 5. A logic algebraized by a quasivariety $\mathrm{K}$ is passively structurally complete iff each existential positive sentence in the first order language of $\mathrm{K}$ holds either in all members of $\mathrm{K}$ or in no nontrivial member of $\mathrm{K}$.

In the proof of Theorem 4, we saw that $\Phi$ holds in $\boldsymbol{B} \in \mathrm{K}$ but not in $\mathbf{2} \in \mathrm{K}$, so a stronger conclusion can be extracted:

Theorem 6. No consistent axiomatic extension of $\mathbf{R}$ is passively structurally complete, except for $\mathbf{C P L}$.

In fact, for $\mathrm{K}$ as in the proof,

$$
p, p \rightarrow(p \rightarrow p),(\neg p \rightarrow p) \rightarrow \neg p / q
$$

is a passive admissible rule of $\vdash^{\mathrm{K}}$, not belonging to $\vdash^{\mathrm{K}}$. For the transition from $\Phi$ to this rule, see [27] or the proof of Theorem $7.3(\Leftarrow)$ in [23].

\section{REFERENCES}

[1] A.R. Anderson, N.D. Belnap, Jnr., 'Entailment: The Logic of Relevance and Necessity, Vol. 1', Princeton University Press, 1975.

[2] C. Bergman, Structural completeness in algebra and logic, in H. Andréka, J.D. Monk and I. Nemeti (eds.), 'Algebraic Logic', Colloquia Mathematica Societatis János Bolyai Vol. 54, North-Holland, Amsterdam, 1991, pp. 59-73.

[3] W.J. Blok, D. Pigozzi, 'Algebraizable Logics', Memoirs of the American Mathematical Society 396, Amer. Math. Soc., Providence, 1989.

[4] S. Burris, H.P. Sankappanavar, 'A Course in Universal Algebra', Graduate Texts in Mathematics, Springer-Verlag, New York, 1981.

[5] P. Cintula, G. Metcalfe, Structural completeness in fuzzy logics, Notre Dame J. Formal Logic 50 (2009), 153-182.

[6] P. Cintula, G. Metcalfe, Admissible rules in the implication-negation fragment of intuitionistic logic, Ann. Pure Appl. Logic 162 (2010), 162-171.

[7] J. Czelakowski, 'Protoalgebraic Logics', Kluwer, Dordrecht, 2001.

[8] J. Czelakowski, W. Dziobiak, Congruence distributive quasivarieties whose finitely subdirectly irreducible members form a universal class, Algebra Universalis 27 (1990), 128-149.

[9] J.M. Dunn, 'The Algebra of Intensional Logics', PhD thesis, University of Pittsburgh, 1966. 
[10] J.M. Dunn, G. Restall, Relevance logic and entailment, in D. Gabbay (ed.), 'Handbook of Philosophical Logic, Vol. 8', 2nd edn., Kluwer, Dordrecht, 2001, pp. 1-128.

[11] W. Dzik, M.M. Stronkowski, Almost structural completeness; an algebraic approach, manuscript.

[12] W. Dziobiak, There are $2^{\aleph_{0}}$ logics with the relevance principle between $R$ and $R M$, Studia Logica 42 (1983), 49-61.

[13] J.M. Font, R. Jansana, D. Pigozzi, A survey of abstract algebraic logic, and Update, Studia Logica 74 (2003), 13-97, and 91 (2009), 125-130.

[14] J.M. Font, G. Rodríguez, Note on algebraic models for relevance logic, Zeitschr. f. math. Logik und Grundlagen der Math. 36 (1990), 535-540.

[15] N. Galatos, P. Jipsen, T. Kowalski, H. Ono, 'Residuated Lattices. An Algebraic Glimpse at Substructural Logics', Studies in Logic and the Foundations of Mathematics 151, Elsevier, 2007.

[16] N. Galatos, J.G. Raftery, A category equivalence for odd Sugihara monoids and its applications, J. Pure Appl. Algebra 216 (2012), 2177-2192.

[17] N. Galatos, J.G. Raftery, Idempotent residuated structures: some category equivalences and their applications, Trans. Amer. Math. Soc. 367 (2015), 3189-3223.

[18] A. Hsieh, J.G. Raftery, Conserving involution in residuated structures, Math. Logic Quarterly 53 (2007), 583-609.

[19] D. Makinson, A characterization of structural completeness of a structural consequence operation, Rep. Math. Logic 6 (1976), 99-102.

[20] R.K. Meyer, J.M. Dunn, E, $R$ and $\gamma$, J. Symbolic Logic 34 (1969), 460-474.

[21] J.S. Olson, J.G. Raftery, Positive Sugihara monoids, Algebra Universalis 57 (2007), 75-99.

[22] J.S. Olson, J.G. Raftery, C.J. van Alten, Structural completeness in substructural logics, Logic J. of the IGPL 16 (2008), 453-495.

[23] J.G. Raftery, Admissible rules and the Leibniz hierarchy, Notre Dame J. Formal Logic, to appear.

[24] V.V. Rybakov, 'Admissibility of Logical Inference Rules', Studies in Logic and the Foundations of Mathematics 136, Elsevier, Amsterdam, 1997.

[25] K. Świrydowicz, A remark on the maximal extensions of the relevant logic $R$, Rep. Math. Logic 29 (1995), 3-13.

[26] K. Świrydowicz, There exist exactly two maximal strictly relevant extensions of the relevant logic $R$, J. Symbolic Logic 64 (1999), 1125-1154.

[27] A. Wroński, Overflow rules and a weakening of structural completeness, in J. SytnikCzetwertyński (ed.), 'Rozważania o Filozofii Prawdziwej. Jerzemu Perzanowskiemu w Darze', Wydawnictwo Uniwersytetu Jagiellońskiego, Kraków, 2009, pp. 67-71.

Department of Mathematics and Applied Mathematics, University of Pretoria, Private Bag X20, Hatfield, Pretoria 0028, South Africa

E-mail address: james.raftery@up.ac.za

Faculty of Mathematics and Computer Science, Adam Mickiewicz UniverSity, Poznań, Poland

E-mail address: swirydow@amu.edu.pl 